\title{
CEREBRAL VENOUS THROMBOSIS AFTER THE FONTAN PROCEDURE
}

William R. Wilson, MD, Georgia E. Greer, MSN, and Joseph D. Tobias, MD, Columbia, Mo

From the Departments of Surgery, Pediatrics, and Anesthesiology, The Children's Hospital, University of Missouri, Columbia.

Received for publication April 23, 1998; accepted for publication May 18, 1998.

Address for reprints: William R. Wilson, MD, MA 312, One Hospital Dr, University of Missouri Health Sciences Center, Columbia, MO 65212.

J Thorac Cardiovasc Surg 1998;116:661-3

Copyright $\odot 1998$ by Mosby, Inc.

$0022-5223 / 98 \$ 5.00+0 \quad \mathbf{1 2 / 5 4 / 9 1 8 6 1}$
The Fontan procedure for repair of single ventricle has become one of the most commonly performed procedures in children with congenital heart disease. Despite improving outcomes, significant neurologic morbidity is seen after this operation. A number of considerations unique to the Fontan procedure and single ventricle physiology have been suggested as predisposing factors for stroke. Sluggish systemic venous flow, arrhythmias, alterations in humoral coagulation systems, and the presence of intracardiac foreign material may predispose to thrombosis. Fenestrations of the atrial tunnel 


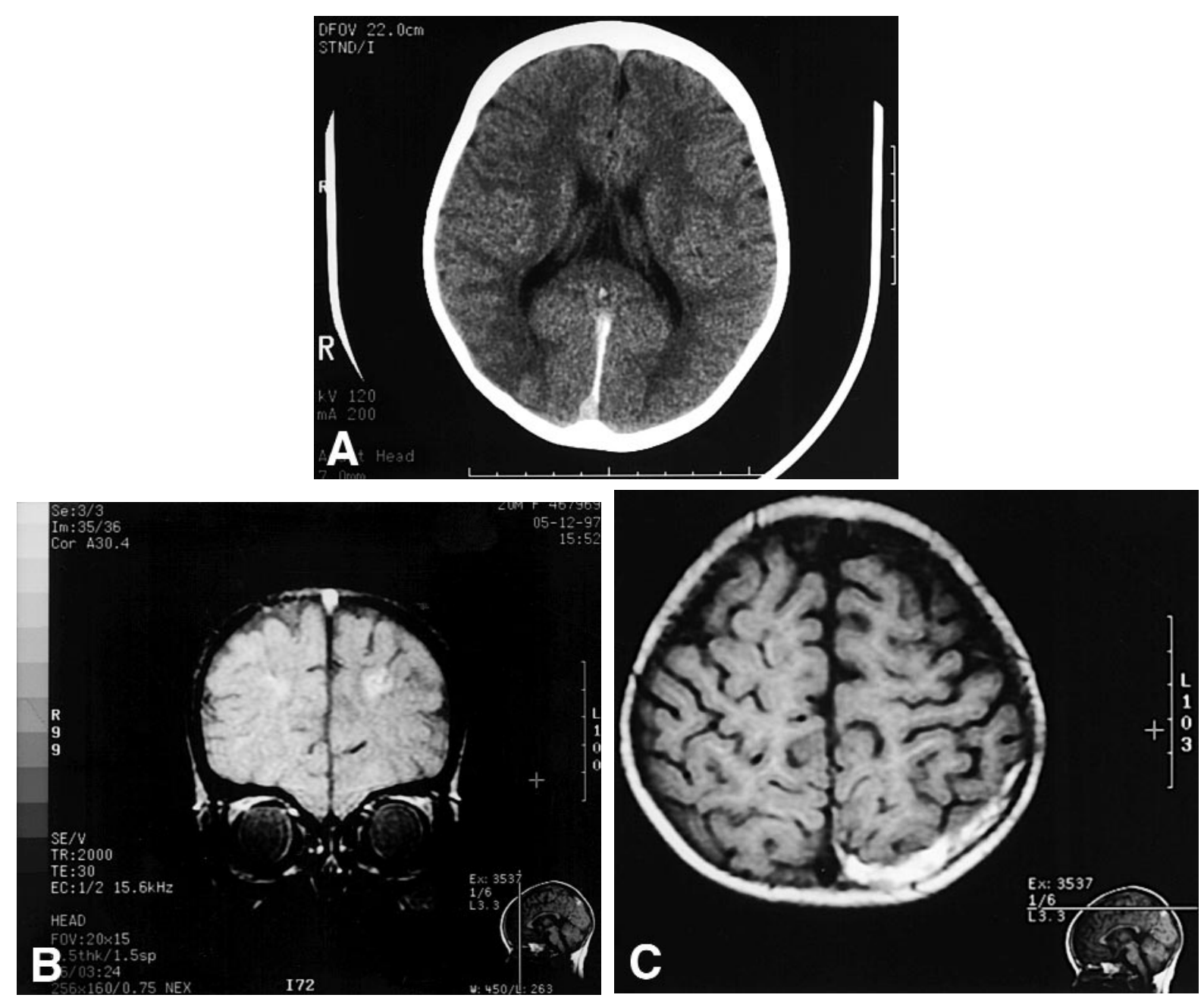

Fig 1. A, Computed tomographic scan without contrast medium shows blood in the straight sinus as dense white line between the occipital hemispheres. B, A magnetic resonance imaging scan in the frontal projection demonstrates an absence of flow void in the midsagittal sinus caused by clot from cerebral venous thrombosis shown as a white opacity. C, Magnetic resonance imaging shows subdural blood in the left occipital area from an associated venous infarct caused by cerebral venous thrombosis

and the stump of a ligated pulmonary artery have been shown to be sources for cerebral embolization. ${ }^{1}$

Cerebral venous thrombosis has been suggested as a potential cause of neurologic injury after the Fontan procedure but has yet to be documented. ${ }^{2}$ Increased systemic venous pressure after the cavopulmonary connection is transmitted to cerebral veins and may lead to stasis and thrombosis. We report a case of central venous thrombosis after a Fontan procedure and suggest that when neurologic problems develop after cavopulmonary connection, this potentially treatable cause of neurologic injury be considered.

Clinical summary. The patient was a 22-month-old girl with aortic atresia, hypoplastic left ventricle, unbalanced atrioventricular canal, common atrium, bilateral superior venae cavae, interrupted inferior vena cava with azygos continuation, polysplenia, and situs inversus who was referred for an elective Fontan procedure. This child's 2 previous cardiac operations consisted of a Norwood stage I repair at 5 days of age and a bilateral bidirectional Glenn shunt at 7 months of age. At the time of the completion Fontan operation, she was receiving no medication, had begun to walk, and was growing well.

The Fontan procedure was performed during a continuous period of cardiopulmonary bypass and moderate hypothermia at $32^{\circ} \mathrm{C}$. Hepatic venous return was baffled through a lateral atrial tunnel to an augmented confluence of the right and left pulmonary arteries. A 4-mm fenestration was created in the lateral tunnel. The patient was weaned from cardiopulmonary bypass with the aid of low-dose intravenous infusions of dobutamine, dopamine, and milrinone.

On the morning of postoperative day 1 , the patient was doing well hemodynamically and was extubated. Six hours later she became somnolent and had focal seizures of the left arm and leg, which were controlled by phenytoin and phenobarbital. The electroencephalogram showed no focal abnor- 
malities but some diffuse symmetric high-voltage delta slowing. A computed tomographic scan (Fig 1, A) showed a venous thrombosis of the straight sinus extending into the midsagittal sinus. Magnetic resonance imaging of the brain (Fig 1, B and $C$ ) confirmed a central venous thrombosis involving the sagittal sinus with a venous infarct of the right occipital region with an associated hematoma. The patient was started on a regimen of aspirin. Over the next 3 days she continued to have a flaccid left arm and leg, but by postoperative day 4 she was much more alert. By postoperative day 10 the patient was without seizures, moving all extremities, and was discharged to her home. Her coagulation profile was normal except for reduced protein $\mathrm{C}$ activity. In the ensuing 7 months since the operation she has had no further seizures, has normal strength bilaterally, and mild hyperreflexia of the left ankle and left knee. She has good ventricular function with normal growth and development.

Discussion. Thromboembolic complications have been noted in a high percentage of patients undergoing a successful Fontan procedure. Rosenthal and coworkers ${ }^{2}$ reported an incidence of $29 \%$, the majority of thromboemboli occurring in the systemic venous circulation and causing no symptoms. The incidence of documented stroke after the Fontan procedure is lower (3\%-9\%). ${ }^{3}$ Abnormalities of the coagulation system may contribute to the high incidence of thromboembolism in patients after the Fontan procedure, most likely as acquired defects resulting from decreased hepatic synthesis. ${ }^{4}$

Cerebral venous thrombosis caused by thrombosis of the major dural sinuses and adjacent dural veins has been reported in a variety of clinical conditions including cyanotic heart disease and hypercoagulable states. The superior sagittal sinus is the most common site, but the thrombosis may involve lateral sinuses or straight sinuses that drain into the internal jugular vein. Thrombosis may extend to cortical veins leading to rupture and a hemorrhagic infarct of the white matter. The clinical course is highly variable, and patients with cere- bral venous thrombosis may have headache or focal physical signs. Up to one third may have focal seizures. Outcomes are also variable, with the majority of patients recovering without sequelae. ${ }^{5}$ Improved imaging techniques make cerebral venous thrombosis more readily identified, and mortality is now estimated at $5.5 \%$. Computed tomographic scan and magnetic resonance imaging may be diagnostic for cerebral venous thrombosis. Current recommendations for treatment include complete anticoagulation with heparin for worsening symptoms in the absence of associated hemorrhagic infarct.

In conclusion, we report a case of cerebral venous thrombosis in a patient after a Fontan procedure. We suggest that this group of patients may be particularly at risk for cerebral venous thrombosis because of sluggish flow in the internal jugular vein, coagulation abnormalities associated with the Fontan physiology, and increased cerebral venous pressure. Investigation of neurologic problems after a Fontan procedure should include magnetic resonance imaging to detect cerebral venous thrombosis as a potential treatable cause.

\section{REFERENCES}

1. Jonas RA. Editorial: Intracardiac thrombus after the Fontan procedure. J Thorac Cardiovasc Surg 1995;110:1502-3.

2. Rosenthal DN, Friedman AH, Kleinman CS, Kopf GS, Rosenfeld LE, Hellenbrand WE. Thromboembolic complications after Fontan operations. Circulation 1995;92(Suppl):II287-93.

3. du Plessis AJ, Chang AC, Wessel DL, Lock JE, Wernosvky G, Newburger JW, et al. Cerebrovascular accidents following the Fontan operation. Pediatr Neurol 1995;12:230-6.

4. Cromme-Dijkhuis AH, Henkens, CM, Bijleveld CM, Hillege HL, Bom VJ, van der Meer, J. Coagulation factor abnormalities as possible thrombotic risk factors after Fontan operations. Lancet 1990;336:1087-90.

5. Ameri A, Bousser MG. Cerebral venous thrombosis. Neurol Clin 1992;10:87-111 